\title{
Circulating levels of angiogenesis-related growth factors in breast cancer: A study to profile proteins responsible for tubule formation
}

\author{
GEMMA A. BARRON ${ }^{1}$, MARIE GOUA ${ }^{2}$, KLAUS W.J. WAHLE ${ }^{3}$ and GIOVANNA BERMANO ${ }^{1}$ \\ ${ }^{1}$ Centre for Obesity Research and Education (CORE), and ${ }^{2}$ School of Pharmacy and Life Sciences (PALS), \\ Robert Gordon University, Aberdeen, Scotland, AB10 7GJ, UK; ${ }^{3}$ School of Medicine, Medical Sciences and Nutrition, \\ University of Aberdeen, Foresterhill, Aberdeen, Scotland, AB25 2ZD, UK
}

Received January 21, 2017; Accepted June 15, 2017

DOI: $10.3892 /$ or.2017.5803

\begin{abstract}
The present study exploited a versatile in vitro endothelial cell/fibroblast co-culture cell system to investigate the association between angiogenesis and breast cancer by comparing the capacity of plasma from women with breast cancer and age-matched controls, to influence tubule formation and modulate angiogenesis in vitro, and to identify plasma circulating factors which might be responsible. Plasma from women with breast cancer $(n=8)$ (added on day 7 after co-culture establishment) significantly increased tubule formation by $57 \%(\mathrm{P}<0.01)$ when compared to cultures grown in culture medium lacking in vascular endothelial growth factor (VEGF) and fetal bovine serum (FBS), whereas plasma from controls $(n=8)$ did not. Higher levels of VEGF, tumour necrosis factor- $\alpha$ (TNF $\alpha$ ) and interleukin (IL)-6, but not leptin, were observed in plasma samples of the breast cancer group compared to the control group ( $\mathrm{n}=20$ in each group). In independent experiments, the effects of VEGF, TNF $\alpha$, IL- 6 and leptin were assessed and it was found that tubule formation was differentially affected whether these inflammatory cytokines or adipokines were added individually or in combination to the co-culture system. Using Proteome Profiler human angiogenesis array kits, 12 out of 55 angiogenesis-related proteins were differentially expressed in plasma from the breast cancer group compared to the control group. Pro-angiogenic proteins included: amphiregulin, artemin, coagulation factor III, fibroblast growth factor (FGF) acidic, GDNF, IL-8, macrophage inflammatory protein (MIP)-1 $\alpha$, platelet derived growth factor-
\end{abstract}

Correspondence to: Dr Giovanna Bermano, Centre for Obesity Research and Education (CORE), Robert Gordon University, Sir Ian Wood Building, Garthdee Road, Aberdeen, Scotland, AB10 7GJ, UK E-mail: g.bermano@rgu.ac.uk

Abbreviations: EC, endothelial cells; HUVEC, human umbilical vein endothelial cells; IL-6, interleukin-6; MMP, matrix metalloproteinases; NHDF, normal human dermal fibroblasts; TNF $\alpha$, tumour necrosis factor- $\alpha$; VEGF, vascular endothelial growth factor

Key words: angiogenesis, breast cancer, co-culture, cytokines
$\mathrm{AB} /$ platelet derived growth factor-BB (PDGF-AB/PDGF-BB) and VEGF, whereas anti-angiogenic proteins were: angiopoietin-2, serpin F1 and serpin B5. In addition, FGF acidic was further identified as differentially expressed, with increased expression, when plasma samples from the normal and cancer groups, which induced an increase in tubule formation, were compared to one another. In conclusion, the present study identified angiogenesis-related proteins circulating in the serum of women with breast cancer that are likely to facilitate the growth and metastasis of breast cancer, in part through their influence on tubule formation, and, therefore, may be potential targets for new cancer therapies.

\section{Introduction}

Breast cancer is the most frequently diagnosed cancer, as well as, the leading cause of death in the female population worldwide (1). The aetiology of breast cancer remains largely unknown, probably due to its multifactorial nature, but several risk factors have been identified (2). These include reproductive and hormonal factors, environmental factors (including lifestyle changes with a higher consumption of animal fat, obesity and limited physical activity) and hereditary factors.

Poor prognosis of breast cancer is strongly related to advanced tumour stage and lymph node spread, although the importance of specific molecular markers has also been investigated (3-5). For a tumour to grow and metastasise it needs to develop its own blood supply and this is achieved through angiogenesis. The surrounding endothelial cells are stimulated to form new blood vessels by growth factors secreted from both the tumour and stroma. The process of angiogenesis is not the result of one single growth factor but rather is dependent on the interaction of multiple proteins with pro- or antiangiogenic properties. In breast cancer, like almost all other solid tumours, growth factors such as vascular endothelial growth factor (VEGF), basic fibroblast growth factor (FGF), hepatocyte growth factor (HGF), platelet derived growth factor (PDGF), monocyte chemoattractant protein (MCP)-1, macrophage inflammatory protein (MIP)-1 $\beta$, interleukin (IL)- 8 and the regulated on activation, normal $T$ cell expressed and secreted (RANTES), are expressed and, depending on 
the subtype of breast cancer, have been identified as negative prognostic factors for patient survival (6-10). These growth factors may be responsible for inducing angiogenesis as they are pro-angiogenic, however, breast cancer cells and tissues can also produce inhibitors of angiogenesis, including thrombospondin, angiostatin and endostatin (11). Thus, it is becoming clear that angiogenesis in cancer progression is the result of a net balance between positive and negative regulators of tubule formation. This evidence directs further investigation into the importance of assessing the roles and levels of angiogenic factors in plasma from women with and without breast cancer. However, despite numerous studies into the detection of circulating biomarkers in breast cancer patients $(9,10,12,13)$, the molecular mechanism(s) by which these circulating factors present in breast cancer may contribute to, and influence angiogenesis, are still poorly understood, suggesting a complex mechanism of interactions. Therefore, the aim of the present study was to assess the ability of circulating factors, present in the plasma from women with breast cancer, to influence tubule formation in an in vitro system (14) and to identify key factors responsible for increased angiogenesis.

\section{Materials and methods}

Materials. All chemicals and cell culture reagents were purchased from Sigma-Aldrich Ltd. (Dorset, UK), unless otherwise stated.

Cell culture. Primary human umbilical vein endothelial cells (HUVEC) and primary normal human dermal fibroblasts (NHDF) were obtained from Clonetics (Lonza, Slough, UK). HUVEC were maintained in endothelial basal medium (EBM-2) supplemented with EGM-2 SingleQuots (i.e., EGM-2 medium) whilst NHDF were maintained in fibroblast basal medium (FBM) supplemented with FGM-2 SingleQuots; all from Clonetics; Lonza (i.e., FGM-2 medium).

The co-culture system was set up using HUVEC (between passages 1-8) and NHDF (between passages 1-12) following the protocol established by Bishop et al (15) and adapted by Barron et al (14). HUVEC and NHDF were mixed and seeded in 24-well plates (Thermo Fisher Scientific Nunc, Loughborough, UK) in EGM-2 medium. Co-cultured cells were incubated for up to 14 days at $37^{\circ} \mathrm{C}$ in a $5 \% \mathrm{CO}_{2}$ in air humidified incubator.

Study subjects. Participants in the study were recruited through the Breast Clinic in Aberdeen Royal Infirmary, Scotland. The study was approved by the Grampian Ethics Committee. Signed informed consent was obtained from each study participant. Venous blood from 40 volunteers was used in this study: 20 women with breast cancer and 20 agematched control individuals. The women with breast cancer had a life-time risk estimated to be double that of the normal female population, based on their history, and screen of no known genetic basis for the development of their breast cancer (e.g., BRCA1 or BRCA2 mutations) (16). The control group included individuals with the normal population risk of breast cancer who did not have breast cancer but were undergoing other forms of breast surgery (16).
Plasma isolation. Plasma was isolated from venous blood from all of the participants using a Histopaque gradient (16). Briefly, $20 \mathrm{ml}$ of blood was layered on top of $20 \mathrm{ml}$ Histopaque-1077 and centrifuged at $800 \mathrm{x}$ g for $30 \mathrm{~min}$ at room temperature. The top layer containing the plasma was collected and stored at $-80^{\circ} \mathrm{C}$ until required.

Plasma samples $(n=40)$ were assayed in duplicate to measure the concentration of VEGF, TNF $\alpha$, IL-6 and leptin (DuoSet ELISA cat. no. DY293B, DY210, DY206 and DY398-05, respectively; R\&D Systems, Abingdon, UK) according to the manufacturer's instructions. These assays have a sensitivity of $\sim 30,15,9$ and $20 \mathrm{pg} / \mathrm{ml}$, respectively, and quantify both the natural and recombinant forms of each protein.

Co-culture treatment with human plasma and combinations of cytokines. The co-culture system was incubated with EGM-2 medium to initiate tubule formation and, 7 days after co-culture establishment, the medium was changed to EGM-2ӨFBS [i.e., EGM-2 medium minus VEGF SingleQuot and 2\% (v/v) fetal bovine serum (FBS) SingleQuot] or EGM-2ӨFBS supplemented with $2 \%$ plasma from a random subset of women with breast cancer $(n=8)$ or control individuals $(n=8)$. The medium was collected every 4 days and replaced with respective treatments.

In order to examine the effects of inflammatory cytokines, the co-culture system was incubated with EGM-2 medium to initiate tubule formation and, 7 days after the co-culture establishment, medium was changed to EGM-2+VEGF, EGM-2 $\Theta$, EGM-2 $\Theta+$ Leptin, or cytokine combinations:

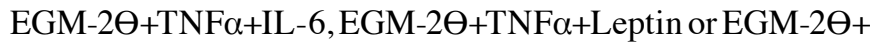
IL-6+Leptin. The medium codes are summarised in Table I. The medium was collected every 4 days and was replaced with respective treatments.

Immunostaining. After 14 days, co-cultures were fixed at room temperature with ice-cold $70 \%(\mathrm{v} / \mathrm{v})$ ethanol and stained with myeloma cell adhesion molecule [MCAM (CD146); specific to HUVEC] to assess the extent of tubule formation as previously described by Barron et al (14). Co-cultures were viewed using an Olympus 1XS1 microscope with Olympus TL4 Light Box Infinity Capture Application version 5.0.0 (Lumenera Corp., Nepean, ON, Canada) with camera model Infinity 2-2c was used for image capture. Three images per well were captured and saved as BMP images of 1616 x 1216 pixels. Illumination was set to give a best contrast between stained tubules and unstained fibroblasts. Tubule formation was defined by total tubule length and quantified using AngioSys ${ }^{\circledR}$ software version 1.0 (TCS Cellworks Ltd., Buckingham, UK).

Proteome Profiler ${ }^{T M}$ Human Angiogenesis Antibody array. The relative expression of 55 angiogenesis-related proteins was determined in human plasma (4 women per group) using a Proteome Profiler ${ }^{\mathrm{TM}}$ Human Angiogenesis Antibody Array according to the manufacturer's instructions (cat. no. ARY007; R\&D Systems). Briefly, after a 1-h membrane blocking step, human plasma $(200 \mu \mathrm{l})$ was pre-incubated with a cocktail of biotinylated detection antibodies $(15 \mu \mathrm{l})$ and added to the membrane before incubating overnight at $4^{\circ} \mathrm{C}$. After a series of washes, the membrane was incubated with streptavidin-horseradish peroxidase (HRP; $2 \mathrm{ml}$ ) for 
Table I. Details of medium employed for co-culture treatments.

\begin{tabular}{|c|c|}
\hline Code & Details \\
\hline EBM-2 & Endothelial basal medium \\
\hline EGM-2 & EBM-2 supplemented with EGM-2 SingleQuots \\
\hline EGM-2Ө & EGM-2 medium minus VEGF SingleQuot \\
\hline EBM-2ӨFBS & EGM-2 medium minus VEGF and FBS SingleQuots \\
\hline EGM-2Ө+Leptin & EGM-2Ө supplemented with leptin \\
\hline EGM-2+VEGF & EGM-2 supplemented with VEGF \\
\hline EGM-2Ө+TNF $\alpha+$ IL-6 & EGM-2 $\Theta$ supplemented with TNF $\alpha$ and IL-6 \\
\hline EGM-2Ө+TNF $\alpha+$ Leptin & EGM-2Ө supplemented with TNF $\alpha$ and leptin \\
\hline EGM-2Ө+IL-6+Leptin & EGM-2 $\Theta$ supplemented with IL-6 and leptin \\
\hline
\end{tabular}

$30 \mathrm{~min}$, before chemiluminescence detection reagents were added in equal volumes for $1 \mathrm{~min}$. The signal was detected by exposing the membrane to CL-XPosure X-ray film (Thermo Fisher Scientific) over a variety of exposure times (1, 3, 5 and $10 \mathrm{~min})$. The light produced at each spot is proportional to the amount of analyte bound and the mean pixel density of the duplicate spots produced on the film was determined using a FUSION FX7 ${ }^{\mathrm{TM}}$ imaging instrument with Fusion 1 and BIO-1D ${ }^{\text {TM }}$ imaging software (PeqLab; VWR International Ltd., Lutterworth, UK).

In addition to the 55 angiogenesis-related proteins, each membrane contained three pairs of positive reference spots and one pair of negative control spots. Following subtraction of the mean pixel density of the negative control spots from all values, the level of each protein was expressed as a ratio relative to the mean of the positive reference spots which were assigned a value of 1 . Relative expression ratios for all 55 angiogenesis-related proteins were compared between plasma from women with breast cancer $(n=3)$ and plasma from control individuals $(n=4)$.

Statistical analysis. Unless otherwise stated, all data are expressed as mean \pm SEM of three independent experiments from different cell passage numbers. Statistical differences were determined by one-way analysis of variance (ANOVA) with Dunnett's multiple comparison t-test (GraphPad Prism). $\mathrm{P}<0.05$ was considered to be statistically significant.

\section{Results}

Effect of human plasma on tubule formation. The effect of human plasma on tubule formation was tested with EGM-2ӨFBS: human plasma replaced FBS (2\% v/v) present in EGM-2 medium minus VEGF. Co-cultures were initially incubated in EGM-2 medium (day 0) and, after co-culture establishment (day 7): EGM-2ӨFBS or EGM-2ӨFBS supplemented with either human plasma from women with breast cancer (cancer group, $n=8$ ) or control (control group, $n=8$ ) individuals was added. These conditions were used to assess the ability of angiogenesis-related growth factors present in human plasma to modulate tubule formation once the angiogenesis process had already started in our in vitro co-culture model.
Table II. Baseline characteristics of control and cancer groups.

\begin{tabular}{lcc}
\hline Characteristics & $\begin{array}{c}\text { Control group } \\
(\mathrm{n}=20)\end{array}$ & $\begin{array}{c}\text { Cancer group } \\
(\mathrm{n}=20)\end{array}$ \\
\hline Age $($ years $)$ & $41.9 \pm 1.3$ & $41.1 \pm 1.2$ \\
VEGF $(\mathrm{pg} / \mathrm{ml})$ & $23.7 \pm 12.2$ & $142.3 \pm 58.2$ \\
TNF $\alpha(\mathrm{pg} / \mathrm{ml})$ & $159.9 \pm 90.6$ & $491.4 \pm 225.8$ \\
IL-6 $(\mathrm{pg} / \mathrm{ml})$ & $43.1 \pm 31.2$ & $153.1 \pm 79.5$ \\
Leptin $(\mathrm{ng} / \mathrm{ml})$ & $15.9 \pm 1.5$ & $17.8 \pm 1.7$ \\
\hline
\end{tabular}

Results are expressed as mean $\pm \mathrm{SEM}$.

Tubules were clearly formed and visualised after 14 days (Fig. 1A) and similar results were obtained in each experiment. The extent of tubule formation was quantified by measuring total tubule length and the percentage change was normalised to EGM-2ӨFBS (Fig. 1B). Tubule formation was significantly increased by $57 \%(\mathrm{P}<0.01)$ when plasma from women with breast cancer was used in the co-culture system compared to EGM-2ӨFBS. Although tubule formation was increased (by $21 \%$ ) with plasma from control individuals; no significant difference was found compared to EGM-2ӨFBS.

Levels of VEGF, TNF $\alpha, I L-6$ and leptin in human plasma. Higher levels of VEGF ( 6-fold increase, $\mathrm{P}=0.053), \mathrm{TNF} \alpha$ ( $\sim 3$-fold increase) and IL-6 ( 3.5-fold increase) were present in plasma samples of the breast cancer group compared to the control group ( $n=20$ in each group), but differences were not statistically significant (Table II) $(\mathrm{P}>0.05)$. No changes in leptin levels were observed between groups (Table II).

Effect of the adipokine leptin and of a combination of inflammatory cytokines/adipokine on tubule formation. We have previously shown that angiogenic factors and inflammatory markers such as VEGF, TNF $\alpha$ and IL-6 play an important role in modulating angiogenesis (14); however, their effects on modulating angiogenesis when used in combination with the adipokine leptin or other inflammatory cytokines have not been previously reported.

The effect of leptin on tubule formation was first examined in the absence of VEGF (i.e., EGM-2 $\Theta$ medium) in a similar 

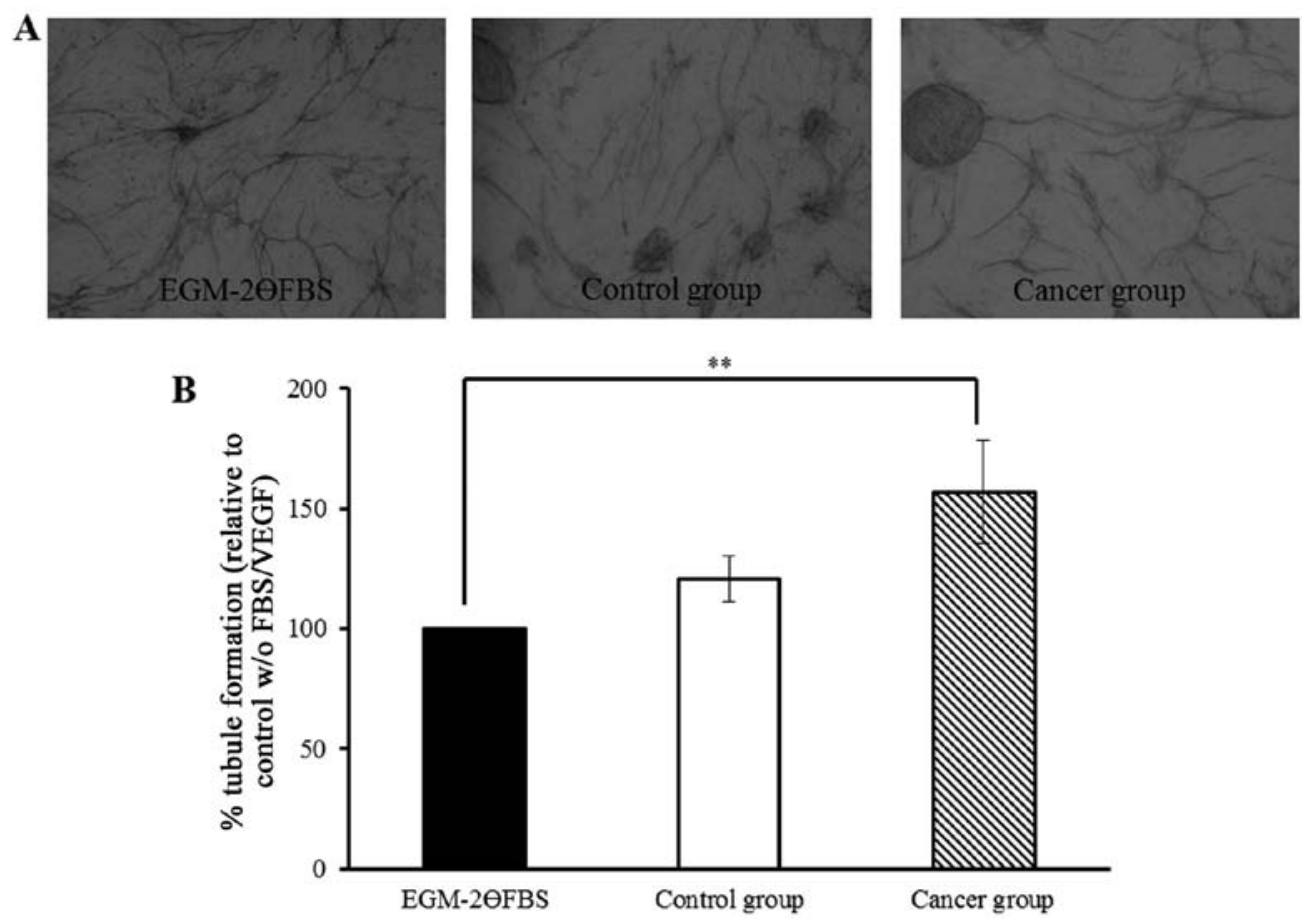

Figure 1. (A) Tubule formation assessed by MCAM staining on day 14 in co-cultures grown in EGM-2ӨFBS, EGM-2ӨFBS supplemented with $2 \%$ plasma from control individuals (control group) or EGM-2ӨFBS supplemented with $2 \%$ plasma from women with breast cancer (cancer group) added on day 7 after co-culture establishment. Medium was changed every 4 days. Images presented are representative of at least 3 independent experiments/plasma samples; within an experiment, each treatment was carried out in duplicate and observed in three randomly chosen fields. Magnification, $\mathrm{x} 40$. (B) Quantification of total tubule length determined using AngioSys software. Plasma was added on day 7. Results are presented as a percentage of EGM-2ӨFBS as mean \pm SEM of at least 3 independent experiments/plasma sample; within each experiment, each treatment was carried out in duplicate; ${ }^{* *} \mathrm{P}<0.01$.

manner to Barron et al (14). Tubule formation was quantified by measuring total tubule length and the percentage change was normalised to EGM-2Ө (Fig. 2A). Incubating co-cultures with leptin (10 nM, added on day 7; EGM-2Ө+leptin) did not alter tubule formation compared to EGM-2 $\Theta$. Whereas, tubule formation was significantly increased $(43 \%)$ when complete EGM-2 medium was added at day 7 compared to EGM-2 $\Theta$, confirming the ability of the co-culture system to further produce tubules with addition of VEGF. However, addition of VEGF (2 ng/ml; EGM-2+VEGF) to complete EGM-2 medium after 7 days did not induce further tubule formation (Fig. 2B), suggesting an inability of the in vitro system to create more new tubules than were normally formed with complete EGM-2 medium as clearly visualised after staining with MCAM on day 14 (Fig. 2C).

Further studies were undertaken to determine the effect of cytokine/adipokine combinations on tubule formation. Co-cultures were initially incubated with EGM-2 medium (day 0) and, after co-culture establishment (day 7), EGM-2 $\Theta$, EGM-2 $\Theta+T N F \alpha+I L-6$, EGM-2 $\Theta+T N F \alpha+L e p t i n$ or EGM-2 $\Theta+$ IL-6+Leptin were added. As combination studies in which cytokines and adipokines are tested together in equal or different concentrations have not been previously carried out, these studies, which use an in vitro system reflecting an in vivo situation where cytokines and adipokines do not exist alone, are therefore very important to identify factors responsible for angiogenesis.

Tubules were clearly formed and visualised after 14 days (Fig. 3A) and similar results were obtained in each experiment. The extent of tubule formation was quantified by measuring total tubule length and the percentage change was normalised to EGM-2Ө (Fig. 3B). TNF $\alpha$ (10 ng/ml) combined with IL-6 $(10 \mathrm{ng} / \mathrm{ml})$ or TNF $\alpha(10 \mathrm{ng} / \mathrm{ml})$ combined with leptin $(10 \mathrm{nM})$ $(\mathrm{P}<0.05)$, reduced tubule formation by 53 and $58 \%$, respectively. IL-6 $(10 \mathrm{ng} / \mathrm{ml})$ combined with leptin $(10 \mathrm{nM})$ did not alter tubule formation compared to EGM-2 $\Theta$ but, tubule formation was increased when a higher concentration of IL-6 $(15 \mathrm{ng} / \mathrm{ml}$, increased by $26 \%)$ or leptin $(15 \mathrm{nM}$, increased by $16 \%$ ) were combined with $\mathrm{TNF} \alpha(5 \mathrm{ng} / \mathrm{ml})$ but, these changes were not statistically significant when compared to EGM-2 $\Theta$. A $114 \%$ increase in tubule formation was observed with IL-6 $(15 \mathrm{ng} / \mathrm{ml})$ combined with leptin $(5 \mathrm{nM})$ but again, this was not statistically significant when compared to EGM-2Ө. No statistically significant differences were observed between the equivalent (white bars) and different (black bars) concentrations of each combination (Fig. 3B). These results indicate that TNF $\alpha$, IL-6 and leptin may differentially modulate tubule formation either added individually or in combination, but the limitation of the co-culture system does not allow this or, alternatively, additional key factors are required.

Comparison of angiogenesis-related protein expression between plasma from women with breast cancer and control group. Although higher levels of VEGF, TNF $\alpha$, IL-6 or leptin were observed in the breast cancer group (Table II), the addition of exogenous VEGF, TNFa, IL-6 or leptin alone (Fig. 2) and Barron et al (14) or in combination (Fig. 3) showed that these molecules are not solely responsible for the increase in tubule formation observed in the breast cancer group (Fig. 1). Proteome profiler human angiogenesis antibody arrays were, 

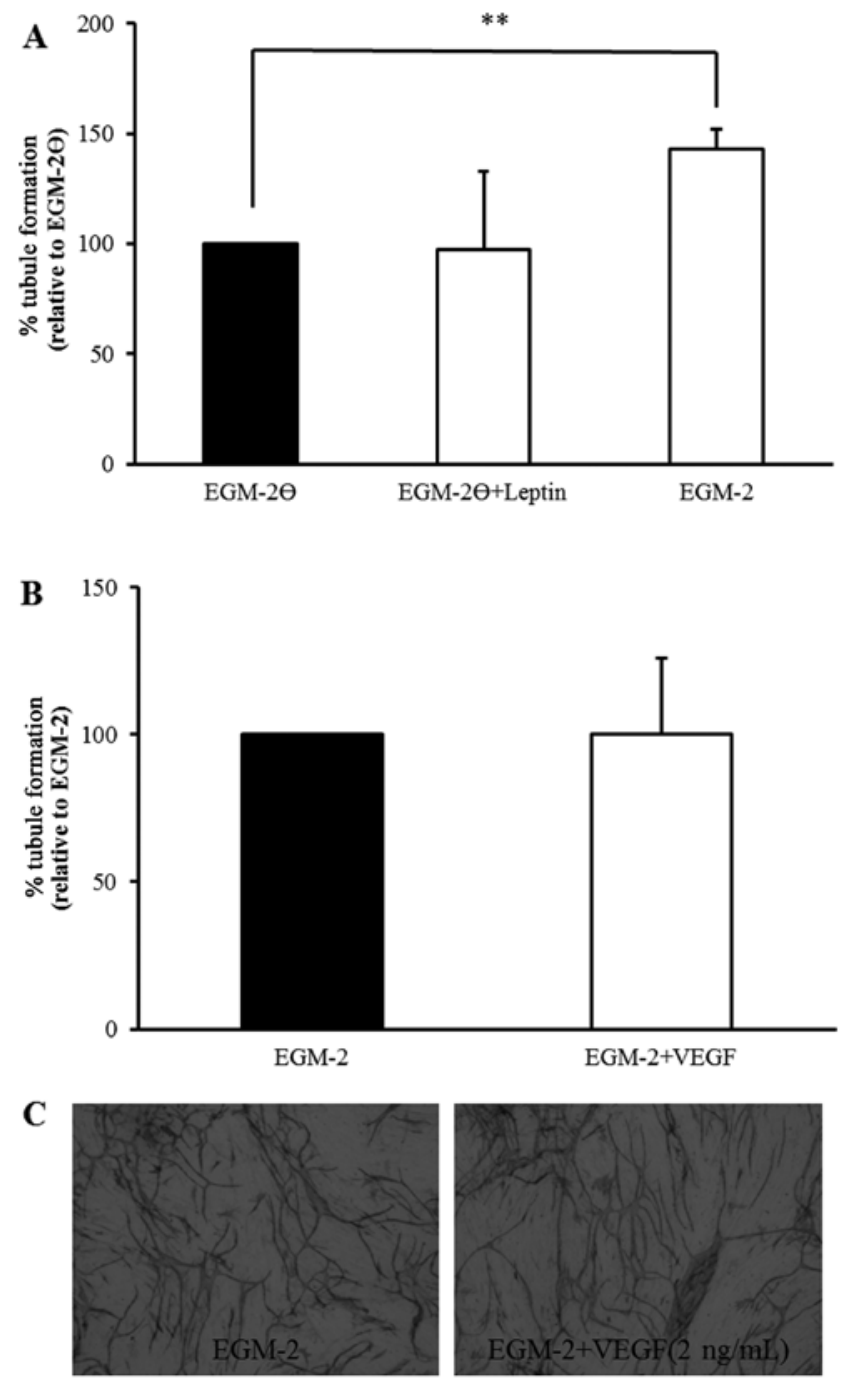

Figure 2. (A) Quantification of total tubule length determined using AngioSys software. Medium changed from EGM-2 on day 7 to EGM-2 $\Theta$, EGM-2 $\Theta+$ Leptin and EGM-2. Medium was changed every 4 days. Results presented as a percentage of EGM- $2 \Theta$ as mean \pm SEM of 3 independent experiments, within each experiment, each treatment was carried out in duplicate; ${ }^{* *} \mathrm{P}<0.01$. (B) Quantification of total tubule length determined using AngioSys software. VEGF $(2 \mathrm{ng} / \mathrm{ml})$ added on day 7 and medium was changed every 4 days. Results presented as a percentage of EGM- 2 as mean \pm SEM of 3 independent experiments, within each experiment, each treatment was carried out in duplicate. (C) Tubule formation assessed by MCAM staining on day 14 in co-cultures grown in EGM-2 and EGM-2+VEGF $(2 \mathrm{ng} / \mathrm{ml})$ added on day 7 after co-culture establishment. Medium was changed every 4 days. Images presented are representative of treatments from 3 independent experiments; within an experiment, each treatment was carried out in duplicate and tubule formation was observed in three randomly chosen fields. Magnification, $\mathrm{x} 40$.

therefore, used to identify differences in protein expression that could be responsible for the increase in tubule formation. Angiogenesis protein profiles in plasma from women with breast cancer $(n=3)$ were compared to profiles from control individuals $(n=4)$ (plasma from one breast cancer individual was not acceptable for protein array analysis and was excluded).

Levels of proteins expressed in plasma from the control group were compared to those in plasma from women with breast cancer, irrespective of whether the plasma sample increased, or not, tubule formation in the in vitro co-culture system. A cut-off of 1.5-fold increase difference and a cut-off
Table III. Relative expression of angiogenic factors which demonstrated no change in protein expression.

\begin{tabular}{|c|c|c|}
\hline Angiogenic factor & $\begin{array}{l}\text { Control group } \\
\qquad(\mathrm{n}=4)\end{array}$ & $\begin{array}{c}\text { Cancer group } \\
(\mathrm{n}=3)\end{array}$ \\
\hline Activin A & 0.07 & 0.07 \\
\hline ADAMTS-1 & 0.08 & 0.10 \\
\hline Angiogenin & 1.14 & 1.23 \\
\hline Angiopoietin-1 & 0.50 & 0.65 \\
\hline Angiostatin/plasminogen & 0.14 & 0.13 \\
\hline CXCL16 & 1.01 & 1.08 \\
\hline DPPIV & 1.07 & 1.04 \\
\hline EGF & 0.14 & 0.12 \\
\hline EG-VEGF & 0.19 & 0.15 \\
\hline Endoglin & 0.97 & 0.89 \\
\hline Endostatin/collagen XVIII & 1.05 & 1.15 \\
\hline Endothelin-1 & 0.54 & 0.67 \\
\hline FGF-4 & 0.10 & 0.09 \\
\hline FGF-7 & 0.06 & 0.07 \\
\hline GM-CSF & 0.12 & 0.09 \\
\hline HB-EGF & 0.19 & 0.16 \\
\hline HGF & 0.14 & 0.12 \\
\hline IGFBP-1 & 1.01 & 1.15 \\
\hline IGFBP-2 & 1.03 & 1.15 \\
\hline IGFBP-3 & 1.02 & 1.12 \\
\hline $\mathrm{IL}-1 \beta$ & 0.09 & 0.07 \\
\hline LAP (TGF- $\beta 1)$ & 0.10 & 0.08 \\
\hline Leptin & 1.00 & 1.07 \\
\hline MCP-1 & 0.06 & 0.06 \\
\hline MMP-8 & 0.97 & 1.06 \\
\hline MMP-9 & 1.03 & 1.04 \\
\hline NRG1- $\beta 1$ & 0.15 & 0.15 \\
\hline Pentraxin 3 (PTX3) & 0.45 & 0.59 \\
\hline PD-ECGF & 0.21 & 0.22 \\
\hline PDGF-AA & 0.60 & 0.78 \\
\hline Platelet factor 4 (PF4) & 1.02 & 1.09 \\
\hline PIGF & 0.11 & 0.11 \\
\hline Prolactin & 0.86 & 0.99 \\
\hline TIMP-4 & 1.07 & 0.73 \\
\hline Thrombospondin-1 & 1.05 & 0.73 \\
\hline Thrombospondin-2 & 0.05 & 0.05 \\
\hline VEGF-C & 0.04 & 0.03 \\
\hline
\end{tabular}

Values (to 2 decimal places) shown are relative expression of angiogenic factor over positive control.

of 0.5-fold decrease difference was used as considered threshold for meaningful physiological changes in protein arrays studies. Thirty-seven proteins in the array showed no change in expression relative to the positive reference spots (value of 1) and were not further examined (Table III). Eighteen angiogenic-related factors demonstrated a level of expression that was 1.5-fold different in either plasma obtained from control or women with cancer. For these 
$\mathbf{A}$
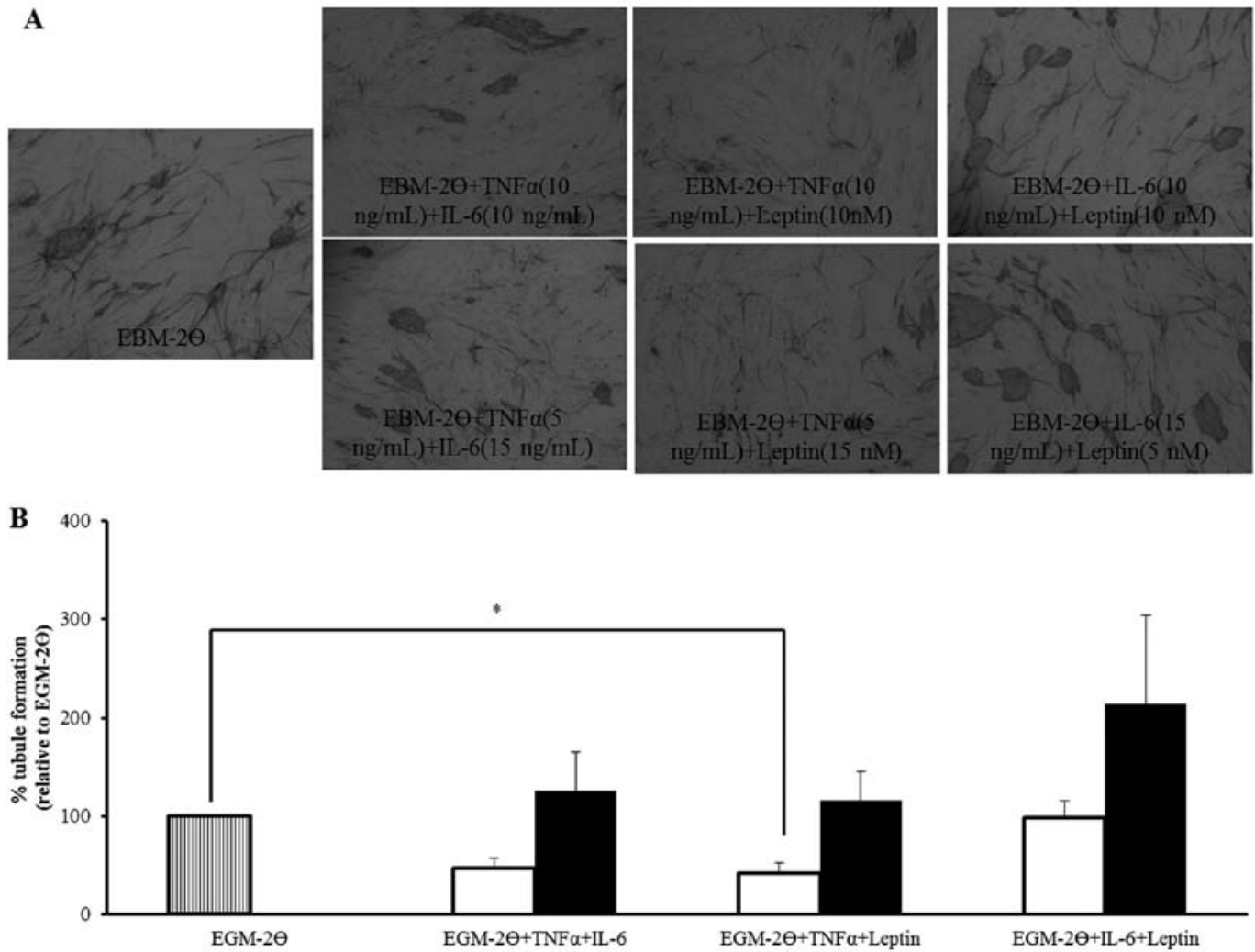

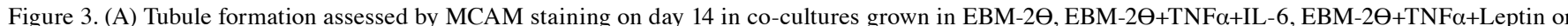
EBM-2Ө+IL-6+Leptin added on day 7 after co-culture establishment. Medium was changed every 4 days. Images presented are representative of at least 3 independent experiments; within an experiment, each treatment was carried out in duplicate and observed in three randomly chosen fields. Magnification, $\mathrm{x} 40$. (B) Quantification of total tubule length determined using AngioSys software. TNFa, IL-6 and leptin added at equivalent concentrations (white bars) (related to top row of images in A) and different concentrations (black bars) (related to bottom row of images in A). Results are presented as a percentage of EGM-2 $\Theta$ (striped bar) as mean \pm SEM of at least 3 independent experiments; within each experiment, each treatment was carried out in duplicate; ${ }^{*} \mathrm{P}<0.05$.

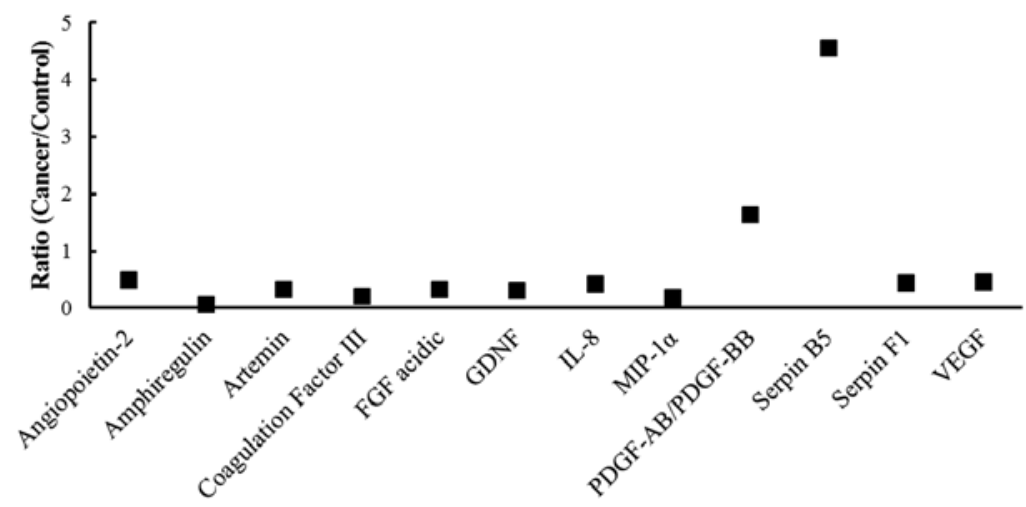

Figure 4. Relative expression of angiogenic factors in breast cancer/control samples.

18 proteins, cancer-to-control ratios were calculated. Overall, 6 proteins were excluded that had ratios between $0.5-1.5$ (i.e. pro-angiogenic: FGF basic, persephin, uPA and vasohibin; anti-angiogenic: serpin E1 and TIMP-1), 10 proteins showed reduced expression (ratio $<0.5$ ) (pro-angiogenic: amphiregulin, artemin, coagulation factor III, FGF acidic, GDNF, IL-8, MIP-1 $\alpha$ and VEGF; anti-angiogenic: angiopoietin-2 and serpin F1) (Fig. 4); whilst, 2 proteins showed increased expression (ratio >1.5): PDGF-AB/PDGF-BB (ratio of 1.64, pro-angiogenic) and serpin $\mathrm{B} 5$ (ratio of 4.56, antiangiogenic) (Fig. 4).
Furthermore, plasma samples, from both the normal and cancer groups, which induced an increase in tubule formation in the in vitro co-culture model, were compared to identify which angiogenesis-related proteins could be responsible for tubule formation. For array analysis, a cut-off of 1.5-fold difference was set as before. In the normal group, 42 out of the 55 angiogenesis-related proteins showed no change in protein expression and were not further examined. Thirteen of the 55 angiogenic-related factors had levels of expression that were 1.5 -fold different. In relation to the expression of these 13 proteins, further analysis was carried out by comparing their 
Table IV. Relative expression of angiogenic factors which demonstrated an increase in tubule formation when plasma from both the control and breast cancer groups was used in the in vitro co-culture system.

\begin{tabular}{|c|c|c|}
\hline $\begin{array}{l}\text { Angiogenic } \\
\text { factor }\end{array}$ & $\begin{array}{l}\text { Increase in } \\
\text { tubule formation } \\
\text { Control group } \\
(\mathrm{n}=2)\end{array}$ & $\begin{array}{l}\text { Increase in } \\
\text { tubule formation } \\
\text { Cancer group } \\
(n=2)\end{array}$ \\
\hline Activin A & $2.28^{\mathrm{c}}$ & $0.36^{\mathrm{b}}$ \\
\hline ADAMTS-1 & - & $0.60^{\mathrm{a}}$ \\
\hline Angiopoietin-1 & $0.64^{\mathrm{a}}$ & $3.54^{\mathrm{c}}$ \\
\hline $\begin{array}{l}\text { Angiostatin/ } \\
\text { plasminogen }\end{array}$ & - & $3.10^{\mathrm{c}}$ \\
\hline Artemin & $1.45^{\mathrm{a}}$ & $3.23^{\mathrm{c}}$ \\
\hline EGF & - & $0.68^{\mathrm{a}}$ \\
\hline EG-VEGF & - & $0.64^{\mathrm{a}}$ \\
\hline Endoglin & - & $0.61^{\mathrm{a}}$ \\
\hline FGF-4 & - & $2.02^{\mathrm{c}}$ \\
\hline FGF acidic & $1.78^{\mathrm{c}}$ & $49.39^{\mathrm{c}}$ \\
\hline GDNF & $2.07^{\mathrm{c}}$ & - \\
\hline GM-CSF & $1.56^{\mathrm{c}}$ & $0.48^{\mathrm{b}}$ \\
\hline HGF & - & $0.62^{\mathrm{a}}$ \\
\hline IL-1 $\beta$ & - & $2.22^{c}$ \\
\hline IL-8 & $0.61^{\mathrm{a}}$ & $1.55^{\mathrm{c}}$ \\
\hline LAP (TGF- $\beta 1)$ & - & $1.49^{\mathrm{a}}$ \\
\hline MIP-1 $\alpha$ & $1.54^{\mathrm{c}}$ & - \\
\hline Pentraxin 3 (PTX3) & - & $0.43^{\mathrm{b}}$ \\
\hline PD-ECGF & - & $0.61^{\mathrm{a}}$ \\
\hline PDGF-AA & $0.61^{\mathrm{a}}$ & $2.54^{\mathrm{c}}$ \\
\hline PDGF-AB/PDGF-BB & $0.37^{\mathrm{b}}$ & $4.31^{\mathrm{c}}$ \\
\hline Persephin & - & $2.02^{c}$ \\
\hline PIGF & - & $1.63^{c}$ \\
\hline Serpin B5 & - & $30.86^{\mathrm{c}}$ \\
\hline TIMP-1 & - & $0.01^{\mathrm{b}}$ \\
\hline TIMP-4 & - & $0.04^{\mathrm{b}}$ \\
\hline Thrombospondin-2 & $8.12^{c}$ & - \\
\hline uPA & $0.61^{\mathrm{a}}$ & $0.11^{\mathrm{b}}$ \\
\hline VEGF & - & $0.24^{\mathrm{b}}$ \\
\hline VEGF-C & $0.57^{\mathrm{a}}$ & $1.54^{\mathrm{c}}$ \\
\hline
\end{tabular}

Values (to 2 decimal places) shown are the ratios of protein expression in plasma samples which induced an increase compared to no change in tubule formation when compared to EBM-2OFBS, for control group and cancer group independently. ${ }^{\text {aAngiogenic-related }}$ proteins that had ratios between $0.5-1.5$; ${ }^{b}$ angiogenic-related proteins that had ratios $<0.5$; and 'angiogenic-related proteins that had ratios $>1.5$.

expression and calculating a ratio between the same proteins in plasma samples which induced an increase-to-no change in tubule formation when compared to EGM-2ӨFBS (Fig. 1). After exclusion of 6 proteins that had ratios between 0.5-1.5 (i.e., pro-angiogenic: angiopoietin-1, artemin, IL-8, PDGF-AA, uPA and VEGF-C), 1 angiogenesis-related protein (i.e. PDGF-AB/PDGF-BB, pro-angiogenic) showed reduced expression (ratio $<0.5$ ) whereas 6 angiogenesis-related proteins (i.e. pro-angiogenic: FGF acidic, GDNF, GM-CSF and MIP-1 $\alpha$; anti-angiogenic: activin A and thrombospondin-2), had increased expression (ratio >1.5) (Table IV).

Conversely, in the cancer group, 28 of the 55 angiogenesisrelated proteins showed no change in protein expression and were not examined further. Twenty-seven of the 55 angiogenesis-related factors identified had levels of expression that were 1.5-fold different (Table IV) and, of these 27 remaining angiogenesis-related proteins, the increase in tubule formation-to-no change in tubule formation ratios was calculated. Overall, after exclusion of 7 angiogenesis-related proteins that had ratios between 0.5-1.5 [i.e. pro-angiogenic: EGF, EG-VEGF, endoglin, HGF, LAP (TGF- $\beta 1$ ), and PD-ECGF; anti-angiogenic: ADAMTS-1], 20 showed differences in levels: the level of expression was reduced (ratio <0.5) for 7 proteins (i.e. pro-angiogenic: GM-CSF, pentraxin 3, uPA and VEGF; anti-angiogenic: activin A, TIMP-1 and TIMP-4) whereas 13 protein levels were increased (ratio $>1.5$ ) (i.e. pro-angiogenic: angiopoietin-1, artemin, FGF acidic, FGF-4, IL-1 $\beta$, IL-8, PDGF-AA, PDGF-AB/PGDF-BB, persephin, PIGF and VEGF-C; anti-angiogenic: angiostatin/plasminogen, and serpin B5) in the cancer group (Table IV).

\section{Discussion}

Angiogenesis is one of the major causes for cancer metastasis. The formation of new blood vessels is essential for cancer growth and metastasis; therefore, the development of angiogenic characteristics is vital in a number of tumour types including breast cancer $(17,18)$. Many studies have measured the production of angiogenesis-related factors in serum from women with breast cancer via ELISA analysis; however, the majority of these have only examined one or two angiogenesisrelated factors, including VEGF $(6,8,19,20)$, basic FGF (6), IL-6 (21), leptin and prolactin (8) and thrombospondin-1 (19). To date, there have only been three studies which have looked at the expression of multiple angiogenic factors in serum from women with breast cancer $(10,12,22)$.

The present study evaluated the effect of factors present in plasma obtained from women with breast cancer compared to age-matched control women in relation to tubule formation, using a versatile in vitro co-culture model established in our laboratory (14) in addition to testing the effect of cytokines/ adipokines added singularly or in combination to the system. Finally it examined the relative expression of 55 angiogenesisrelated proteins in a subset of plasma samples from cancer and control individuals in relation to tubule formation. This study therefore provides additional information on proteins differentially expressed and on the importance of a balance between pro-angiogenic/anti-angiogenic factors in tubule formation.

The in vitro co-culture system used to study tubule formation has been optimised so that molecules of interest could be added 7 days after initiation of tubule formation to make it relevant when considering breast tumour progression and metastasis. By using plasma samples, it was found that tubule formation in vitro was significantly increased $(\mathrm{P}<0.01)$ after incubation with plasma from women with breast cancer $(n=8)$. Indeed, a higher level of VEGF was present in the breast cancer 
group compared to the control group. However, not all individual plasma samples increased tubule formation, which could be due to low/absent levels of VEGF but also, to higher levels of the anti-angiogenic growth factor, $\mathrm{TNF} \alpha$. As inflammatory cytokines such as TNFa (23) and IL-6 (unpublished data) and, the adipokine leptin have been shown, in vitro, to play a putative role in breast cancer progression (24), it is important to investigate the effect that these exogenous cytokines/adipokines might have on angiogenesis in isolation, but also to study their effect when present in combination as these growth factors do not exist in isolation in human plasma. Using our in vitro co-culture cell model, it was found that leptin did not alter tubule formation when VEGF was lacking but, addition of complete EGM-2 medium supplemented with VEGF did further increase tubule formation. These results are in contrast with the pro-angiogenic effect of leptin $(10,100$ and $1000 \mathrm{ng} / \mathrm{ml})$ on endothelial tube formation previously described in single-cell conditions using HUVECs and a matrigel matrix (25-27) or, HUVECs and a collagen I matrix (28); suggesting that the system used for studying angiogenesis in vitro might be critical even if the use of in vitro co-culture systems are more closely related to in vivo conditions.

This study is also the first to use an in vitro endothelial cell/fibroblast co-culture cell system to investigate the effect of combining exogenous cytokines/adipokines. Results from this study revealed that when $\mathrm{TNF} \alpha$ was combined with either IL-6 or leptin, tubule formation was reduced but, not to the same extent as TNF $\alpha$ alone as it stopped tubule formation (14); therefore, pro-angiogenic IL- 6 and leptin may counteract the anti-angiogenic effect of TNF $\alpha$, but not completely. An increase in tubule formation was observed when IL-6 was combined with leptin. These observations further indicate that angiogenesis is modulated by specific pro- and anti-angiogenic inflammatory markers. More specifically, TNF $\alpha$, IL-6 and leptin differentially affect tubule formation either individually or in combination. Thus, other angiogenesis-related proteins may be involved. Since angiogenesis is a complex process that involves many proteins; angiogenesis protein arrays were used to assess the expression of a large group of proteins. Several angiogenesisrelated proteins (both pro- and anti-angiogenic) were observed to have altered expression in the plasma from women with breast cancer which may correlate with the increase in tubule formation and therefore, may be involved in breast tumour progression and metastasis. Accordingly, angiogenesis in breast cancer is regulated by a net balance between pro- and anti-angiogenic proteins. In the present study, 12 out of 55 angiogenesis-related proteins analysed had altered expression in the breast cancer group compared to the control group; with 10 proteins observed to have reduced expression (pro-angiogenic: amphiregulin, artemin, coagulation factor III, FGF acidic, GDNF, IL-8, MIP-1 $\alpha$ and VEGF; anti-angiogenic: angiopoietin- 2 and serpin F1); whilst, 2 proteins showed increased expression: PDGF-AB/ PDGF-BB (ratio of 1.64, pro-angiogenic) and serpin B5 (ratio of 4.56, anti-angiogenic). To the authors' knowledge, this is the first study to directly examine angiogenesis-related protein expression and tubule formation via a co-culture cell system in vitro using human plasma samples. However, a study by Morelli et al (19), investigated the in vitro effect of sera from patients with breast or gastrointestinal cancers on HUVEC proliferation using a colourimetric assay. Morelli et al (19) observed that sera from $19 \%$ (15 of 78) of breast cancer patients induced growth of HUVECs whilst, sera from 5\% (4 of 78) of breast cancer patients inhibited endothelial cell proliferation. Furthermore, when sera stimulated endothelial growth, it was correlated with high levels of VEGF detected by ELISA whilst, when sera inhibited endothelial cell growth, it was correlated with high levels of soluble thrombospondin revealed by western blot analysis (19). These results show that individual variations of angiogenesis-related proteins are evident in specific cohorts of study volunteers and could depend on disease stage, subtype and treatment. In relation to this, we recognise a limitation of this study to analyse the effects on tubule formation in the co-culture stystem in function of disease stage and subtype: no treatment differences were present as samples were collected just after diagnosis of breast cancer and before treatment commenced.

The present study focused on the identification of factors which drive angiogenesis, however, it also highlighted the importance of the fine balance between pro- and anti-angiogenic factors present in cancer patients' plasma and driving angiogenesis. To date, anti-angiogenic drugs for cancer treatment have been of relative success and have predominantly targeted the expression/binding of pro-angiogenic factors such as VEGF (29). Our data emphasise the need for development of drug therapies which target, at the same time, not only the expression of pro-angiogenic factors but also affect the expression of some anti-angiogenic factors as it is the result of their differential expression which affects angiogenesis and cell proliferation.

In conclusion, many angiogenesis-related proteins may be involved in the growth, spread and progression of breast tumours. This study identified potential key factors which might be responsible to drive angiogenesis in breast cancer and, once their role has been defined further, they might provide targets for future clinical therapies. The identified factors have been shown to be relevant in other malignancies including gastrointestinal (19) and laryngeal cancer (30), confirming their potential importance in breast cancer.

\section{Acknowledgements}

The authors would like to thank Tenovus Scotland and Robert Gordon University (Faculty of Health and Social Care) for financial support. The authors would like to thank Professor S.D. Heys from the University of Aberdeen for facilitating collection of the volunteers' samples.

\section{References}

1. DeSantis CE, Lin CC, Mariotto AB, Siegel RL, Stein KD, Kramer JL, Alteri R, Robbins AS and Jemal A: Cancer treatment and survivorship statistics, 2014. CA Cancer J Clin 64: 252-271, 2014.

2. DeBruin LS and Josephy PD: Perspectives on the chemical etiology of breast cancer. Environ Health Perspect 110 (Suppl 1): 119-128, 2002.

3. Weigelt B, Peterse JL and van 't Veer LJ: Breast cancer metastasis: Markers and models. Nat Rev Cancer 5: 591-602, 2005

4. Taneja P, Maglic D, Kai F, Zhu S, Kendig RD, Fry EA and Inoue K: Classical and novel prognostic markers for breast cancer and their clinical significance. Clin Med Insights Oncol 4: 15-34, 2010.

5. Yersal O and Barutca S: Biological subtypes of breast cancer: Prognostic and therapeutic implications. World J Clin Oncol 5: 412-424, 2014. 
6. Dirix LY, Vermeulen PB, Pawinski A, Prové A, Benoy I, De Pooter C, Martin M and Van Oosterom AT: Elevated levels of the angiogenic cytokines basic fibroblast growth factor and vascular endothelial growth factor in sera of cancer patients. Br J Cancer 76: 238-243, 1997.

7. Relf M, LeJeune S, Scott PA, Fox S, Smith K, Leek R, Moghaddam A, Whitehouse R, Bicknell R and Harris AL: Expression of the angiogenic factors vascular endothelial cell growth factor, acidic and basic fibroblast growth factor, tumor growth factor $\beta-1$, platelet-derived endothelial cell growth factor, placenta growth factor, and pleiotrophin in human primary breast cancer and its relation to angiogenesis. Cancer Res 57: 963-969, 1997.

8. Coskun U, Günel N, Toruner FB, Sancak B, Onuk E, Bayram O, Cengiz O, Yilmaz E, Elbeg S and Ozkan S: Serum leptin, prolactin and vascular endothelial growth factor (VEGF) levels in patients with breast cancer. Neoplasma 50: 41-46, 2003.

9. Chavey C, Bibeau F, Gourgou-Bourgade S, Burlinchon S, Boissière $\mathrm{F}$, Laune $\mathrm{D}$, Roques $\mathrm{S}$ and Lazennec $\mathrm{G}$ : Oestrogen receptor negative breast cancers exhibit high cytokine content. Breast Cancer Res 9: R15-R26, 2007.

10. Gonzalez RM, Daly DS, Tan R, Marks JR and Zangar RC: Plasma biomarker profiles differ depending on breast cancer subtype but RANTES is consistently increased. Cancer Epidemiol Biomarkers Prev 20: 1543-1551, 2011.

11. Castañeda-Gill JM and Vishwanatha JK: Antiangiogenic mechanisms and factors in breast cancer treatment. J Carcinog 15 $1-39,2016$

12. Li L, Chen L, Zhang W, Liao Y, Chen J, Shi Y and Luo S: Serum cytokine profile in patients with breast cancer. Cytokine 89: 173-178, 2016.

13. Rovati B, Mariucci S, Delfanti S, Grasso D, Tinelli C, Torre C, De Amici M and Pedrazzoli P: Simultaneous detection of circulating immunological parameters and tumor biomarkers in early stage breast cancer patients during adjuvant chemotherapy. Cell Oncol (Dordr) 39: 211-228, 2016.

14. Barron GA, Bordet E, Goua M and Bermano G: Modulation of angiogenesis by inflammatory markers and the role of matrix metalloproteinases in an endothelial cell/fibroblast co-culture system. Curr Angiogenes 3: 152-163, 2014.

15. Bishop ET, Bell GT, Bloor S, Broom IJ, Hendry NF and Wheatley DN: An in vitro model of angiogenesis: Basic features. Angiogenesis 3: 335-344, 1999.

16. Bermano G, Smyth E, Goua M, Heys SD and Wahle KW: Impaired expression of glutathione peroxidase- 4 gene in peripheral blood mononuclear cells: A biomarker of increased breast cancer risk. Cancer Biomark 7: 39-46, 2010.

17. Schneider BP and Miller KD: Angiogenesis of breast cancer. J Clin Oncol 23: 1782-1790, 2005.

18. Carmeliet P and Jain RK: Molecular mechanisms and clinical applications of angiogenesis. Nature 473: 298-307, 2011.
19. Morelli D, Lazzerini D, Cazzaniga S, Squicciarini P, Bignami P, Maier JA, Sfondrini L, Ménard S, Colnaghi MI and Balsari A: Evaluation of the balance between angiogenic and antiangiogenic circulating factors in patients with breast and gastrointestinal cancers. Clin Cancer Res 4: 1221-1225, 1998.

20. Gisterek I, Matkowski R, Lacko A, Sedlaczek P, Szewczyk K, Biecek P, Halon A, Staszek U, Szelachowska J, Pudelko M, et al: Serum vascular endothelial growth factors a, $\mathrm{C}$ and $\mathrm{d}$ in human breast tumors. Pathol Oncol Res 16: 337-344, 2010.

21. Zhang GJ and Adachi I: Serum interleukin-6 levels correlate to tumor progression and prognosis in metastatic breast carcinoma. Anticancer Res 19 (2B): 1427-1432, 1999.

22. Georgiou GK, Igglezou M, Sainis I, Vareli K, Batsis H, Briasoulis E and Fatouros M: Impact of breast cancer surgery on angiogenesis circulating biomarkers: A prospective longitudinal study. World J Surg Oncol 11: 213-221, 2013.

23. Weichhaus M, Broom I and Bermano G: The molecular contribution of TNF- $\alpha$ in the link between obesity and breast cancer. Oncol Rep 25: 477-483, 2011.

24. Weichhaus M, Broom J, Wahle K and Bermano G: Leptin inhibits proliferation of breast cancer cells at supraphysiological concentrations by inhibiting mitogen-activated protein kinase signaling. Oncol Lett 8: 374-378, 2014.

25. Rodrigues S, Van Aken E, Van Bocxlaer S, Attoub S, Nguyen QD, Bruyneel E, Westley BR, May FE, Thim L, Mareel M, et al: Trefoil peptides as proangiogenic factors in vivo and in vitro: Implication of cyclooxygenase-2 and EGF receptor signaling. FASEB J 17: 7-16, 2003

26. Garonna E, Botham KM, Birdsey GM, Randi AM, Gonzalez-Perez RR and Wheeler-Jones CP: Vascular endothelial growth factor receptor- 2 couples cyclo-oxygenase-2 with proangiogenic actions of leptin on human endothelial cells. PLoS One 6: e18823, 2011.

27. Dubois V, Delort L, Billard H, Vasson MP and CaldefieChezet F: Breast cancer and obesity: In vitro interferences between adipokines and proangiogenic features and/or antitumor therapies? PLoS One 8: e58541, 2013.

28. Ferla R, Bonomi M, Otvos L Jr and Surmacz E: Glioblastomaderived leptin induces tube formation and growth of endothelial cells: Comparison with VEGF effects. BMC Cancer 11: 303-314, 2011.

29. Nielsen DL, Andersson M, Andersen JL and Kamby C: Antiangiogenic therapy for breast cancer. Breast Cancer Res 12: 209-224, 2010.

30. Korampalli TS, Green V, Greenman J and Stafford ND: Protein profiling of angiogenesis-related growth factors in laryngeal carcinoma: pattern of protein expression in relation to tumour progression. Int J Oncol 39: 1033-1039, 2011. 